\title{
Cuando la tecnología se usa para enriquecer el mundo*
}

\author{
Alfredo ÁlVAREZ ÁlVAREZ \\ Universidad de Alcalá \\ a.alvarezalvarez@uah.es \\ http://orcid.org/0000-0003-3845-8917
}

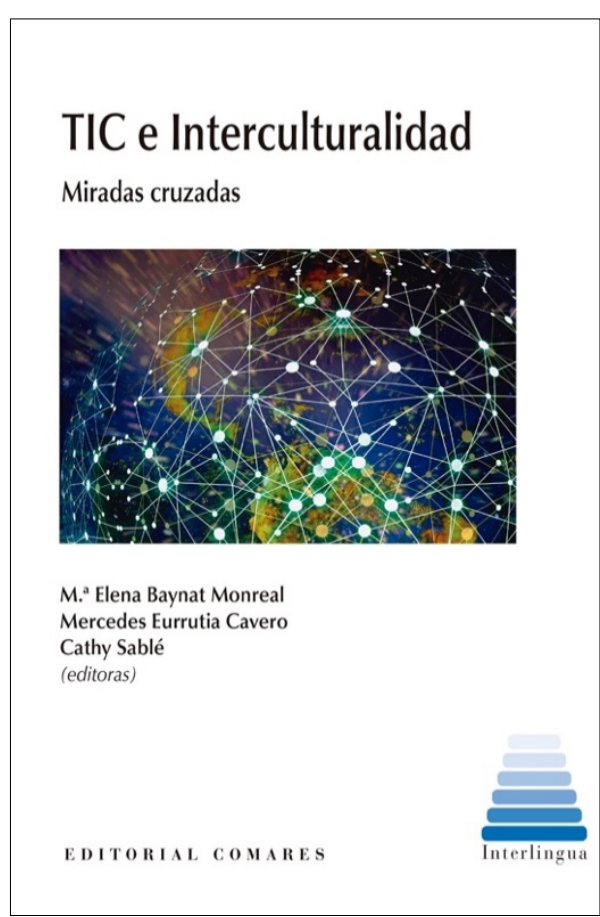

El título del libro que se presenta, TIC e interculturalidad: miradas cruzadas, resulta revelador. Las TIC, por una parte, abren espacios al mundo, lo ensanchan, contribuyendo con ello a redimensionarlo y, en la medida de lo posible, a comprenderlo. Esta dimensión no puede considerarse sin la interculturalidad, sin esa relación de intercambio y comunicación igualitarios entre comunidades diferentes. Cabría tal vez hablar de hiperculturalidad (ByunChul: 2018) o, simplemente, de la caída del horizonte que ha traído la globalización, proceso este que no hubiera sido posible sin la contribución de la tecnología. En todo caso, es tarea de la universidad apostar por el conocimiento e intentar ofrecer a la sociedad lo que Aristóteles denominaba su telos, su propósito, su fin, su objetivo, que no es otro que el de la verdad (Haidt \& Lukianoff: 2019). Y esta, con más intensidad que en otros momentos de la historia, se ve ahora amenazada de múltiples formas, algunas de las cuales llevan el sello tecnológico. Hoy vemos cómo tecnología y mentira se asocian peligrosamente en una ecuación que es imperativo resolver. ¿Significa eso que las TIC muestran el lado perverso del ser humano? No necesariamente, pero nos fotografían como somos, a veces en un espejo deformante, lo que hace que sea tarea de todos contribuir a convertir los espacios

\footnotetext{
* Acerca del volumen colectivo editado por Ma Elena Baynat Monreal, Mercedes Eurrutia Cavero \& Cathy Sablé, TIC e interculturalidad: miradas cruzadas (Granada, Editorial Comares, col. «Interlingua», 2020, 370 p. ISBN: 978-84-1369-029-2).
} 
virtuales en entornos respirables, donde se valore la verdad pura y simple, ya que globalización y diversidad no se excluyen entre sí, ni se ven necesariamente confrontadas; antes al contrario, representan un enriquecimiento en el que las fronteras tradicionales significan más bien poco.

La obra en cuestión aborda un amplio y atractivo conjunto de temas trenzados entre las tecnologías, es decir, la comunicación y el conocimiento, y la relevancia que representa la cultura en la diversidad tomando como objeto de estudio los estudiantes, entendidos como el grupo de población sobre el que dirigir atentas miradas. Está estructurada en catorce capítulos que analizan diversos aspectos del binomio Tic e interculturalidad, y lo hacen desde una multiplicidad de visiones integradoras que van a contribuir a abrir espacios para la reflexión y, al tiempo, para la acción en el entorno de la clase.

En el primero de ellos, Juan Miguel Aguado Terrón analiza algunos aspectos de la comunicación digital en relación con el proceso de «emocionalización» del discurso público a través de diversos vectores, entre los que se pueden considerar la importancia de la información personal como activo económico en el activo digital, la expansión de la cadena de valor de los contenidos mediante la integración de la actividad social de los usuarios en la creación de valor, y la consolidación de los nuevos escenarios de consumo del contenido articulados en torno a la formas tecnológicamente mediadas de interacción social. Resulta evidente que la sociedad se encuentra en un claro proceso de cambio en el que Aguado distingue tres vectores: la «ratificación» de la vida social con la integración de la inteligencia artificial como proceso, la consolidación del trabajo afectivo como mercancía y como fuerza de trabajo gratuita y, finalmente, la mencionada «emocionalización» del discurso público, como resultado de la centralidad de las redes afectivas entendidas como escenarios de puesta en escena de la identidad.

En el segundo capítulo, Sophie Aubin analiza la cuestión de las denominadas brechas digitales e interculturalidad en didáctica de las lenguas culturas. En unos momentos en los que nos sentimos subyugados por la tecnología, es habitual que la mayoría de los estudios sobre esta y su integración en la enseñanza contemplen dicha integración con un lógico optimismo, considerando los indiscutibles beneficios que la herramienta está aportando a la enseńanza-aprendizaje de la lengua extranjera. Sin embargo, Aubin identifica hasta cinco tipos de brechas que están produciéndose en la actualidad; si bien con el fin de superar esas desigualdades propone que, para superar el desafío digital de la didáctica lenguas culturas, habrán de unir esfuerzos los investigadores en interculturalidad y los especialistas en el tratamiento de las brechas digitales.

Los capítulos tercero ( $\mathrm{M}^{\mathrm{a}}$ Elena Baynat Monreal) y decimoprimero (Cathy Sablé) tienen en común el trabajo sobre redes sociales y por esta razón preferimos agruparlos, ya que ambos casos son coincidentes en el análisis del aprendizaje de lenguas mediante la intervención de una red social-Facebook en el primer caso, YouTube en 
el segundo-, lo que plantea el espacio interrelacional como entorno de aprendizaje. En un caso (Facebook), este se favorece mediante un trabajo en colaboración colectiva, lo que mejora y contribuye a la relación interpersonal, conectando a los estudiantes entre sí con independencia de sus diferentes nacionalidades, ideologías o costumbres. En el otro (YouTube), se plantea la pertinencia del uso de clips publicitarios procedentes de la mencionada red, entendiendo que estos no son sino una expresión de lo cultural, pues cada vídeo está incuestionablemente inserto en un tiempo y en un espacio sociológico determinado.

Los capítulos cuatro (María Loreto Cantón Rodríguez), nueve (Mercedes López Santiago) y decimocuarto (Francisca Suau-Jiménez) se consagran a la tríada turismo-interculturalidad-tecnología, proponiendo diferentes y valiosas miradas. En el primer caso, se ha estudiado la transformación del turista tipo tradicional en turista 4.0 o «e-turista», surgido del mundo tecnológico y capaz de organizar sus propios viajes sin la mediación de intermediarios del sector, como fue costumbre y necesidad hasta los inicios del siglo XXI. En dicha transformación han tenido un papel protagónico los blogs, herramientas que, en principio, no estuvieron pensadas específicamente para esta tarea, pero que están cumpliendo su cometido, asumiendo al mismo tiempo una función informativa en la que se plantea, de hecho -como hace Suau-Jiménez, en su caso con las reseñas online de viajeros-, la confrontación entre comunicación y persuasión que puede presentarse a través de la subjetividad de las diferentes propuestas. En el capítulo nueve, López presenta un estudio léxico contrastivo sobre el campo nocional de la interculturalidad, a partir de documentos publicados en la web oficial de turismo de Madrid, en español y en su versión en lengua francesa; con ello se completa el marco turismo-interculturalidad, analizando cómo se interpretan la cultura y la interculturalidad en el ámbito institucional.

En el capítulo cinco, Odile Challe se ocupa del componente intercultural y la empresa, analizando las intersecciones entre fronteras y culturas; espacios que-como señala la autora- han demostrado en Europa una importante debilidad en el caso de la crisis sanitaria de la COVID 19, donde se ha podido comprobar como a pesar del acuerdo Schengen no se ha logrado mantener las fronteras abiertas. En la dimensión lingüística se plantea, igualmente, la cuestión de los límites y la necesidad de gestionar el aspecto intercultural en el mundo interconectado de las empresas, especialmente en aquellas que presentan mayores dimensiones, de las que se espera sean capaces de manejarse tanto en lo global como en lo local.

En el capítulo sexto (Mohamed El-Madkouri Maataoui) se analizan algunos fraseologismos franceses, explorando su traducción mediante cuatro programas de traducción automática: Google.translate, DeepL, Bing y Tradukka. Parece obvio que la de traductor es una profesión llamada en un futuro cercano a profundas transformaciones $y$, en ese camino, programas como los mencionados, aún con sus actuales imperfecciones, parecen destinados a desempeñar tal vez un papel, si bien aún por determinar. Tal 
como El-Madkouri concluye, los mencionados programas están aún lejos de la perfección en la aprehensión del aspecto intercultural de la traducción, lo que no resta un ápice de interés a un análisis detallado de cada una de las aplicaciones en las que pueden observarse tanto sus fortalezas como sus debilidades.

Los capítulos séptimo (Mercedes Eurrutia Cavero) y decimosegundo (Antonia Sánchez Villanueva) se ocupan de distintos lenguajes en entornos virtuales. En el primer caso, Eurrutia analiza el fenómeno pic speech o imagen conversacional a la luz de los nuevos comportamientos sociales de los jóvenes. Cierto es que el uso de las tecnologías ha acarreado modificaciones en las formas de comunicar, especialmente entre este grupo social; formas que podrían encuadrarse en tres vertientes (Álvarez: 2016): de un lado, las nuevas pautas de comunicación que se observan entre los jóvenes, de otro la búsqueda de formas de expresión hiperrápidas y, por último, el desbordamiento del concepto de intimidad entendido a la manera tradicional, que ha llevado a recuperar el de extimidad, propuesto en su día por Lacan, para calificar el fenómeno; lo que a su vez, como apunta Eurrutia, ha dado lugar al concepto de e-reputación. Este, por cierto, no se vehicula del mismo modo en todas las culturas en la medida en que los emojis no significan lo mismo en cada una de ellas. Sánchez Villanueva, por su parte, se ocupa en el mencionado capítulo decimosegundo de los clichés en los medios de comunicación como factor de manipulación. En él constata que los clichés nunca han desaparecido de los mass media, si bien en la actualidad, por influencia de las redes sociales y otros espacios digitales, han dado un salto cualitativo y cuantitativo acuñándose, por cierto, nuevas locuciones que tienen en el espacio Internet un caldo de cultivo idóneo para crecer y desarrollarse. Entre las causas que identifica la autora para la pervivencia de clichés y estereotipos, se encuentran la exigencia de rapidez, la inmediatez, la competencia y la escasez de recursos que sufren los medios de comunicación, situación que ayuda poco a vencerlos.

En el capítulo octavo (Alison Gouvees-Hayward) se trata la cuestión de la interculturalidad en espacios intermedios reales, imaginarios o virtuales. Estos, con la difuminación de las fronteras y la creciente interconexión están, a juicio de la autora, convirtiéndose en una nueva norma. Más específicamente, en el ámbito de la educación, el concepto de espacio intermedio se presenta generalmente como una oportunidad positiva para el aprendizaje intercultural; en particular, en el ámbito de la enseñanza superior, entendiendo que estos espacios intermedios pueden ser al mismo tiempo sinónimo de libertad pero también de alienación.

En el capítulo décimo, Leyre Ruiz de Zarobe se plantea la enseñanza de la interculturalidad a través de la pragmática, partiendo de que la adquisición de una lengua extranjera, si se pretende lograr una competencia comunicativa eficaz, corre paralela a la adquisición de una competencia intercultural en esa lengua. Su propuesta pasa por el análisis de una serie de recursos disponibles en Internet; en concreto, las webs CARLA, Spanish Pragmatics: Dancing with Words y Slaitresearch, que se han mostrado 
instructivas para la enseñanza de la pragmática y eficaces en su transmisión de la interculturalidad, favoreciendo, a juicio de la autora, la creación de una verdadera conciencia intercultural en el estudiante.

Finalmente, en el capítulo decimotercero, Mercedes Sanz Gil se ocupa de los MOOC como recurso para comprender la interculturalidad de lo cotidiano, ya que considera que la interculturalidad se juega en lo cotidiano y este desempeńa también un papel en la aprehensión e interpretación de la interculturalidad. Analiza con detenimiento tres MOOC por su fuerte componente intercultural: Comprender las dimensiones interculturales en la cotidianidad (Universidad de los Andes), Intercultural skills (Asociación Erasmus + Open Virtual Mobility) y, por último, Puertas abiertas: curso de español para necesidades inmediatas (Grupo ATLAS - UNED). En el análisis constata Sanz que, si bien en los tres está presente una sensibilidad hacia la hibridez lingüística como seńa de identidad, aunque también como herramienta para facilitar la comprensión entre los ciudadanos, los aspectos cotidianos se ven tratados desde la reflexión, con mente abierta y receptiva para el encuentro y el entendimiento.

El libro TIC e interculturalidad: miradas cruzadas ofrece, pues, un amplio abanico de opciones desde las que contemplar las diferentes y fructíferas intersecciones entre tecnologías e interculturalidad, lo que contribuirá, ciertamente, a ensanchar las posibilidades de integración de estas herramientas tan útiles y ya imprescindibles en el ámbito de la enseńanza y aprendizaje de las lenguas.

\section{REFERENCIAS BIBLIOGRÁFICAS}

ÁlVAREZ ÁlVAREZ, Alfredo (2016): «Lenguajes en red: nuevos soportes, viejos usos», in Manuel Linares Herrera, José Díaz Cuesta \& $\mathrm{M}^{\mathrm{a}}$ Elena del Valle Mejías (eds.), Innovación universitaria: digitalización 2.0 y excelencia en contenidos. Madrid, McGrawHill Education, 25-34.

BYUNG-CHUl, Han (2018): Hiperculturalidad. Traducción de Florencia Gaillour. Barcelona, Herder editorial.

HAIDT, Jonathan \& Greg LUKIANOFF (2019): La transformación de la mente moderna. Traducción de Verónica Puertollano. Barcelona, Editorial Planeta. 